\title{
Discovery and Rational Design of pteridin-7(8H)-one-based Inhibitors Targeting FMS-like Tyrosine Kinase 3 (FLT3) and Its Mutants
}

Deheng Sunł, Yu Yang \$, Jiankun Lyu \$, Wei Zhou, Wenlin Song, Zhenjiang Zhao, Zhuo Chen*, Yufang $\mathrm{Xu}^{*}$, and Honglin $\mathrm{Li}^{*}$

State Key Laboratory of Bioreactor Engineering, Shanghai Key Laboratory of New Drug Design, School of Pharmacy, East China University of Science \& Technology, Shanghai 200237, China.

$\$$ Authors contributed equally to this work

* To whom correspondence should be addressed. Email: chenzhuo@ecust.edu.cn, yfxu@ecust.edu.cn, hlli@ecust.edu.cn

\section{Content}

In vitro enzymatic activity assay

Figure S1. 2D histogram of selectivity profile data for compound 1 versus a panel of 26 kinase targets.S4

Docking Results. S4

Table S1 Docking scores (Gscores), FLT3 $\mathrm{IC}_{50} \mathrm{~s}, \mathrm{pIC}_{50} \mathrm{~s}$ and QplogKhsas for all compounds. S4

Figure S2. The predicted binding modes of compound 23(A), 22(B), 11(C) and 1(D). . S6

Figure S3. Linear relationship between the $\mathrm{FLT}_{3} \mathrm{pIC}_{50} \mathrm{~s}$ and Gscores for (A) all pteridin-7(8H)-one compounds and three docked FLT3 type I inhibitors, and (B) compounds 1, 7, 11, 12, 18, 19, 22 and 23.. S7 
Figure S4. The predicted binding modes of compound 23(A), 28(C) and 29(B) with P-loop hidden for clarity......

Figure S5. Compound $\mathbf{3 1}$ docked into the active site of the FLT3 homology model. The protein is shown as cyan surface, while the two residues of the solvent slot are highlighted in red color. S8

Kinase Profiling Assay and Binding Constants $\left(\mathrm{K}_{\mathrm{D}} \mathrm{S}\right)$ Assay. ................................................................ 88

Table S2 Kinase Selectivity of compound 31 against a panel of 468 kinases....................................... S9

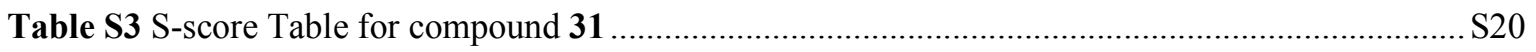

The in vitro enzymatic selectivity against FLT3 over EGFR. ................................................................. S2

Table S4 In vitro enzymatic inhibitory activities and enzyme selectivity of Pteridin-7(8H)-one compounds.

Compound 23 inhibited FLT3 auto-phosphorylation and the phosphorylation of downstream signaling

effectors STAT5, ERK, and AKT in 2 hours S23

Figure S6. After 2 hours treatment, compound 23 inhibited FLT3 auto-phosphorylation and the phosphorylation of downstream signaling effectors STAT5, ERK, and AKT in MV4-11 cells...... S23

Compound 23 induce apoptosis and cell cycle arrest in MV4-11 after $48 \mathrm{~h}$ treatment.

Figure S7. After 48 hours treatment, compound 23 induced dose-dependent $G_{0} / G_{1}$ phase cell cycle arrest (A) and apoptosis (B) in MV4-11 cells. S24 In vivo effecacy of compound $\mathbf{3 1}$ against MV4-11 tumor xenografts via intraperitoneal administration. .. S25

Figure S8. In vivo effects of compound $\mathbf{3 1}$ against MV4-11 tumor xenografts via intraperitoneal administration. (A) Compound $\mathbf{3 1}$ was administered intraperitoneally at $25,50 \mathrm{mg} / \mathrm{kg} / \mathrm{d}$. Data are shown as mean \pm SEM. (B) Body weight change in mouse xenograft model for each daily dosing group.............. S25

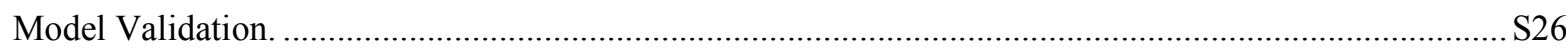

Figure S9. Ramachandran plot of the FLT3 homology model in an active conformation..................... S26

Figure S10. Active and inactive conformations of FLT3. The template protien of FLT3 homology model in an active conformation is a c-KIT kinase (PDB ID: 1PKG). S27

Figure S11. The predicted binding modes, the corresponding FLT3 $\mathrm{IC}_{50}$ values and binding energies of the three known FLT3 type I inhibitors. P-loop is hid for clarity. The $\mathrm{IC}_{50}$ values of CEP-701 and PCK-412 from Blood 2009, 114 (14): 2984-2992. The $\mathrm{IC}_{50}$ value of Crenolanib from Blood 2014,123(1):94-100.. S27 


\section{In vitro enzymatic activity assay}

Compound 1 was screened against 25 kinases in mobility shift assay and BRAF kinase in BRAF assay by Shanghai ChemPartner Co., Ltd.. The inhibition values were obtained in duplicate for 2 concentrations $(10 \mu \mathrm{M}$ and $1 \mu \mathrm{M})$ against the 26 kinases, and staurosporine was used as the reference compounds.

\section{A. Mobility shift assay.}

\section{Kinase reaction.}

Dilute the tested compound to 50X of the final desired highest inhibitor concentration in reaction by $100 \%$ DMSO. Transfer $100 \mathrm{ul}$ of the compound dilution to a well in a 96-well plate. Add $100 \mu \mathrm{l}$ of $100 \%$ DMSO to two empty wells for no compound control and no enzyme control in the same 96-well plate. Mark the plate as source plate. Transfer $10 \mu$ of compound from source plate to a new 96-well plate as the intermediate plate. Add $90 \mu \mathrm{l}$ of 1x kinase buffer (50 mM HEPES, 0.0015\% Brij-35, $10 \mathrm{mM} \mathrm{MgCl} 2,2 \mathrm{mM}$ DTT, $\mathrm{pH}=7.5$ ) to each well of the intermediate plate. Mix the compounds in intermediate plate for $10 \mathrm{~min}$ on shaker. Transfer $5 \mu \mathrm{l}$ of each well from the 96-well intermediate plate to a 384-well plate in duplicates. Add $10 \mu \mathrm{l}$ of $2.5 \mathrm{x}$ enzyme solution to each well of the 384-well assay plate. Incubate at room temperature for $10 \mathrm{~min}$. Add $10 \mu \mathrm{l}$ of $2.5 \mathrm{x}$ peptide solution to each well of the 384 -well assay plate. Incubate at $28 \mathrm{C}$ for specified period of time. Add $25 \mu \mathrm{l}$ of stop buffer (100 mM HEPES, 0.015\% Brij-35, 0.2\% Coating Reagent \#3, $50 \mathrm{mM}$ EDTA, $\mathrm{pH}=7.5)$ to stop reaction. Collect data on Caliper.

\section{Curve fitting.}

Copy conversion data from Caliper program and convert conversion values to inhibition values.

Percent inhibition $=(\max -$ conversion $) /(\max -\min ) * 100$. "max" stands for DMSO control; "min" stands for low control.

\section{B. BRAF assay.}

\section{Kinase assay.}

Dilute the compound to $100 \mathrm{X}$ of the final desired highest inhibitor concentration in reaction by $100 \%$ DMSO. Transfer 100ul of this compound dilution to a well in a 96-well plate. Add $100 \mu 1$ of $100 \%$ DMSO to two empty wells for no compound control and no enzyme control in the same 96-well plate. Mark the plate as source plate. Transfer $4 \mu \mathrm{l}$ of compound from source plate to a new 96-well plate as the intermediate plate. Add $96 \mu \mathrm{l}$ of $1 \mathrm{x}$ kinase buffer $(50 \mathrm{mM}$ HEPES, $10 \mathrm{mM} \mathrm{MgCl} 2,1 \mathrm{mM}$ EGTA, $0.01 \%$ BRIJ-35) to each well of the intermediate plate. Mix the compounds in intermediate plate for $10 \mathrm{~min}$ on shaker. Transfer $2.5 \mu \mathrm{l}$ of each well from the 96-well intermediate plate to a 384-well plate in duplicates. Add $5 \mu \mathrm{l}$ of 2x kinase solution (BRAF $3.5 \mathrm{nM}$ ) to each well of the assay 
plate, except for control wells without enzyme (add $5 \mu 1$ of $1 x$ kinase buffer instead). Shake the plate. Add $2.5 \mu \mathrm{l}$ of $4 \mathrm{x}$ substrate solution $(0.2 \mu \mathrm{M}$ Fluorescein-MAP2K1 and $0.5 \mu \mathrm{M}$ ATP) to each well of the assay plate to start reaction. Shake the plate. Cover the assay plate and incubate at room temperature for 1 hour. Add $10 \mu \mathrm{l}$ of $2 \mathrm{x}$ detection solution (Antibody $2 \mathrm{nM}$ and EDTA $10 \mathrm{mM}$ ) in antibody dilution buffer to each well of the assay plate to stop the reaction. Mix briefly with Centrifuge and incubate at least 30 minutes before reading on a plate reader for fluorescence. Collect data on Envision with excitation at 340nm and emission at $520 \mathrm{~nm}$ and $495 \mathrm{~nm}$.

\section{Curve fitting.}

Copy values of RFU from Envision program. Calculate the ratio of RFU $520 \mathrm{~nm} / \mathrm{RFU} 495 \mathrm{~nm}$ and then convert ratio values to percent inhibition values.

Percent inhibition $=(\max -$ sample ratio $) /(\max -\min ) * 100$. "max" means the ratio DMSO control and "min" means the ratio of no enzyme control.

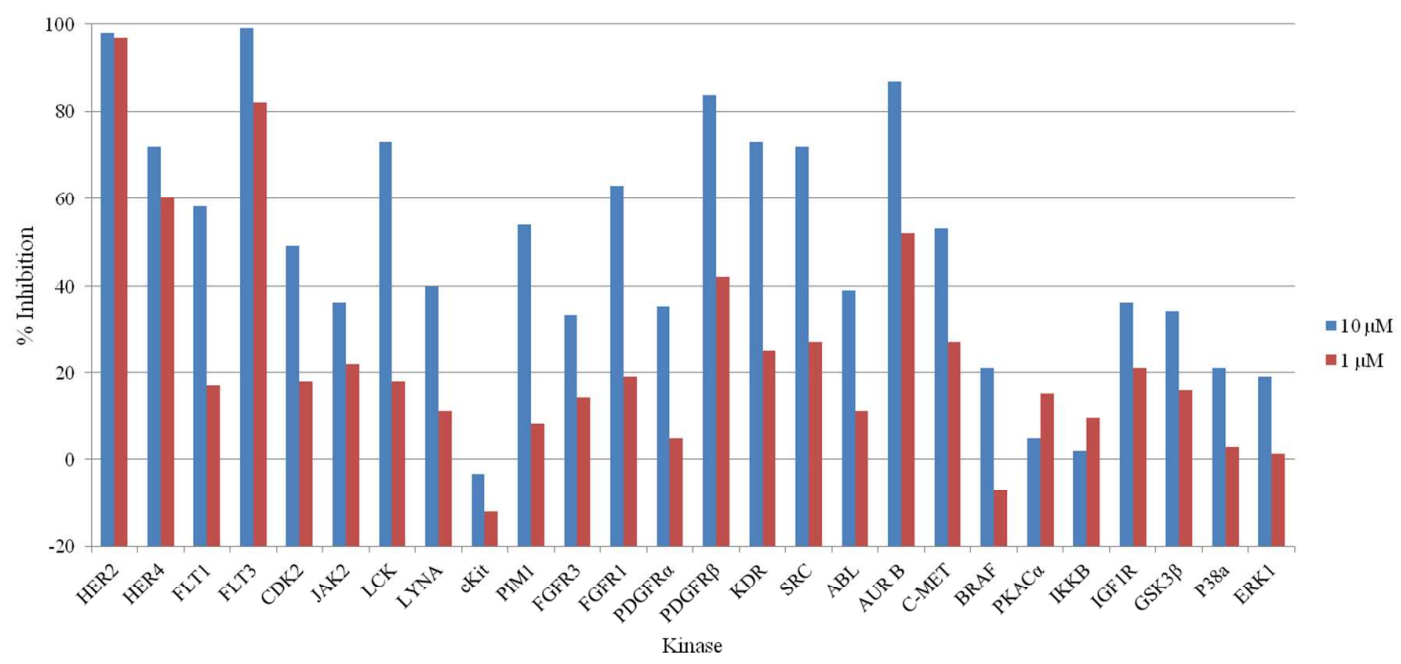

Figure S1. 2D histogram of selectivity profile data for compound 1 versus a panel of 26 kinase targets.

\section{Docking Results.}

Table S1 Docking scores (Gscores), FLT3 $\mathrm{IC}_{50} \mathrm{~S}, \mathrm{pIC}_{50} \mathrm{~s}$ and QplogKhsas for all compounds.

\begin{tabular}{ccccc}
\hline Cpd. & Gscore $(\mathrm{kcal} / \mathrm{mol})$ & ${\text { FLT3 } \mathrm{IC}_{50}(\mathrm{nM})}$ & $\mathrm{pIC}_{50}{ }^{\mathrm{a}}$ & QplogKhsa $^{\mathrm{b}}$ \\
\hline $\mathbf{1}$ & -7.015 & 312 & 6.505845 & 0.116 \\
$\mathbf{2}$ & -6.566 & 1528 & 5.815877 & 0.282 \\
$\mathbf{3}$ & -9.367 & 1056 & 5.976336 & 0.398 \\
$\mathbf{4}$ & -8.100 & 1747 & 5.757707 & 0.261
\end{tabular}




\begin{tabular}{|c|c|c|c|c|}
\hline 5 & -6.374 & 2958 & 5.529002 & -0.212 \\
\hline 6 & -8.081 & 5449 & 5.263683 & -0.162 \\
\hline 7 & -7.914 & 1869 & 5.728391 & -0.060 \\
\hline 8 & -8.725 & 2920 & 5.534617 & -0.056 \\
\hline 9 & -8.866 & 3589 & 5.445027 & 0.044 \\
\hline 10 & -8.018 & 1136 & 5.944622 & -0.013 \\
\hline 11 & -9.760 & 47 & 7.327902 & -0.015 \\
\hline 12 & -8.611 & 108 & 6.966576 & -0.070 \\
\hline 13 & -9.03 & 165 & 6.782516 & -0.056 \\
\hline 14 & -8.947 & 384 & 6.415669 & 0.043 \\
\hline 15 & -9.645 & 173 & 6.761954 & -0.125 \\
\hline 16 & -8.469 & 4413 & 5.355266 & -0.018 \\
\hline 17 & -9.392 & 184 & 6.735182 & -0.029 \\
\hline 18 & -9.638 & 34 & 7.468521 & -0.139 \\
\hline 19 & -9.223 & 29 & 7.537602 & -0.139 \\
\hline 20 & -6.082 & 1317 & 5.880414 & 0.103 \\
\hline 21 & -8.549 & 153 & 6.815309 & -0.033 \\
\hline 22 & -10.848 & 6.13 & 8.21254 & 0.049 \\
\hline 23 & -10.633 & 8.74 & 8.058489 & 0.048 \\
\hline 24 & -9.570 & 127 & 6.896196 & -0.113 \\
\hline 25 & -9.696 & 255 & 6.59346 & -0.485 \\
\hline 26 & -9.093 & 22.2 & 7.653647 & -0.046 \\
\hline 27 & -8.904 & 32 & 7.49485 & -0.378 \\
\hline 28 & -9.587 & 37.2 & 7.429457 & 0.143 \\
\hline 29 & -10.643 & 8.2 & 8.086186 & 0.364 \\
\hline 30 & -9.546 & 347 & 6.459671 & -0.020 \\
\hline 31 & -11.049 & 1.56 & 8.806875 & 0.166 \\
\hline 32 & -11.843 & 3.19 & 8.496209 & 0.058 \\
\hline 33 & -10.118 & 5.24 & 8.280669 & 0.137 \\
\hline 34 & -8.983 & 27.7 & 7.55752 & -0.061 \\
\hline 35 & -9.924 & 35.8 & 7.446117 & 0.064 \\
\hline P-701 & -10.509 & 2.6 & 8.585027 & $0.555\left(>99 \%{ }^{c}\right)$ \\
\hline $\mathrm{AC} 220$ & $\mathrm{ND}^{\mathrm{d}}$ & $4.2^{\mathrm{e}}$ & 8.376751 & $0.476(99 \%$ f $)$ \\
\hline
\end{tabular}

\footnotetext{
${ }^{\text {a. }} \mathrm{pIC}_{50}=-\log _{10}\left(\mathrm{IC}_{50}\right), \mathrm{IC}_{50} \mathrm{~s}$ are specified in units of molar concentration.
}

b. Prediction of binding to human serum albumin by QikProp 3.2. The value range is from -1.5 to 1.5. The higher value of QplogKhsa indicates the higher degree of plasma protein binding; The lower value of QplogKhsa 
indicates the lower degree of plasma protein binding.

c. The experimental plasma protein binding in the parentheses is reported by Ma, Hayley S., et al. Cancer Res. 2014, 74(18): 5206-5217.

d. Not Determined

e. Data from Blood 2009, 114 (14): 2984-2992.

f. The experimental plasma protein binding in the parentheses is reported by Zarrinkar, Patrick P., et al. Blood 2009, 114(14): 2984-2992.

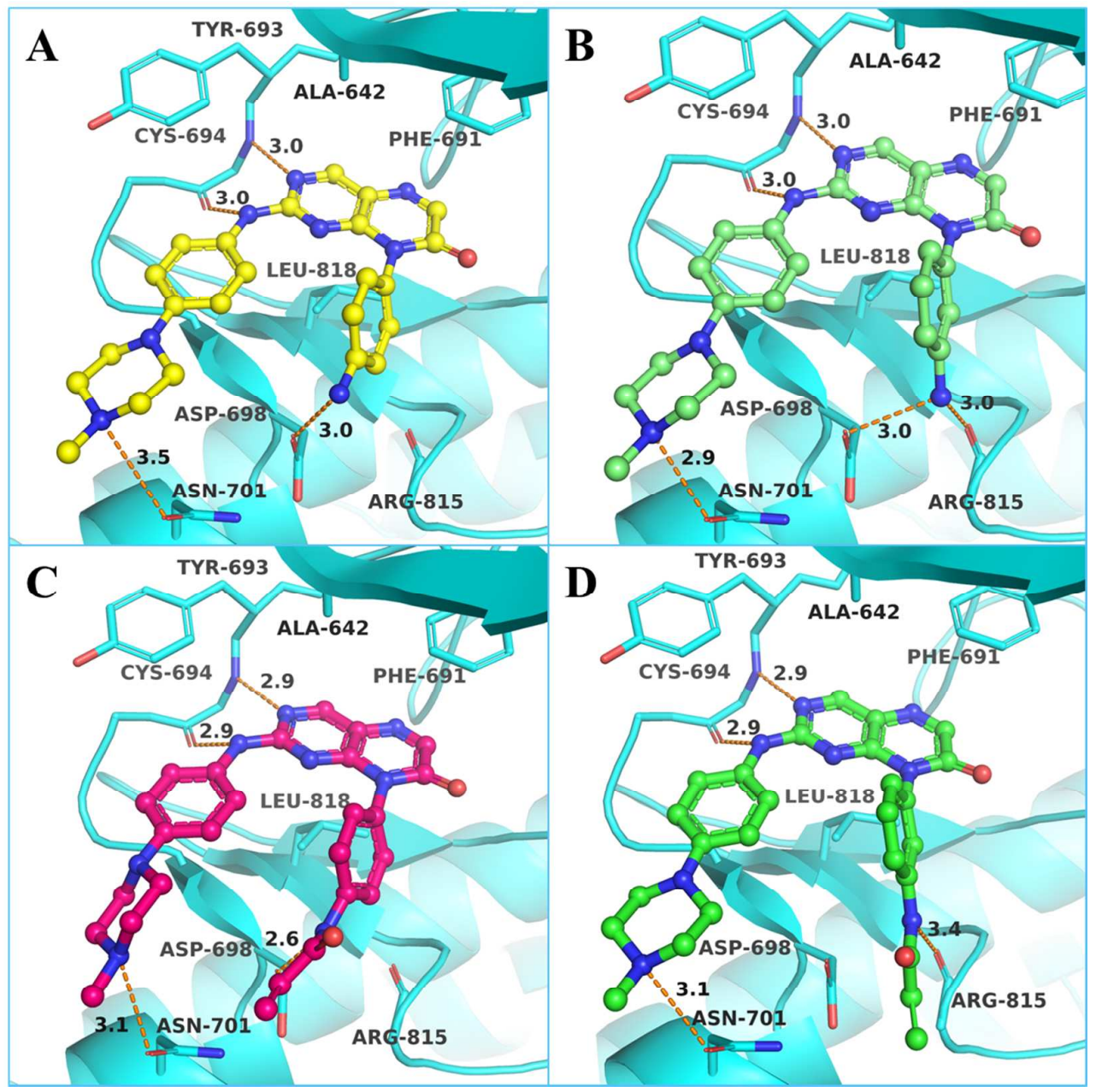

Figure S2. The predicted binding modes of compound 23(A), 22(B), 11(C) and 1(D). 


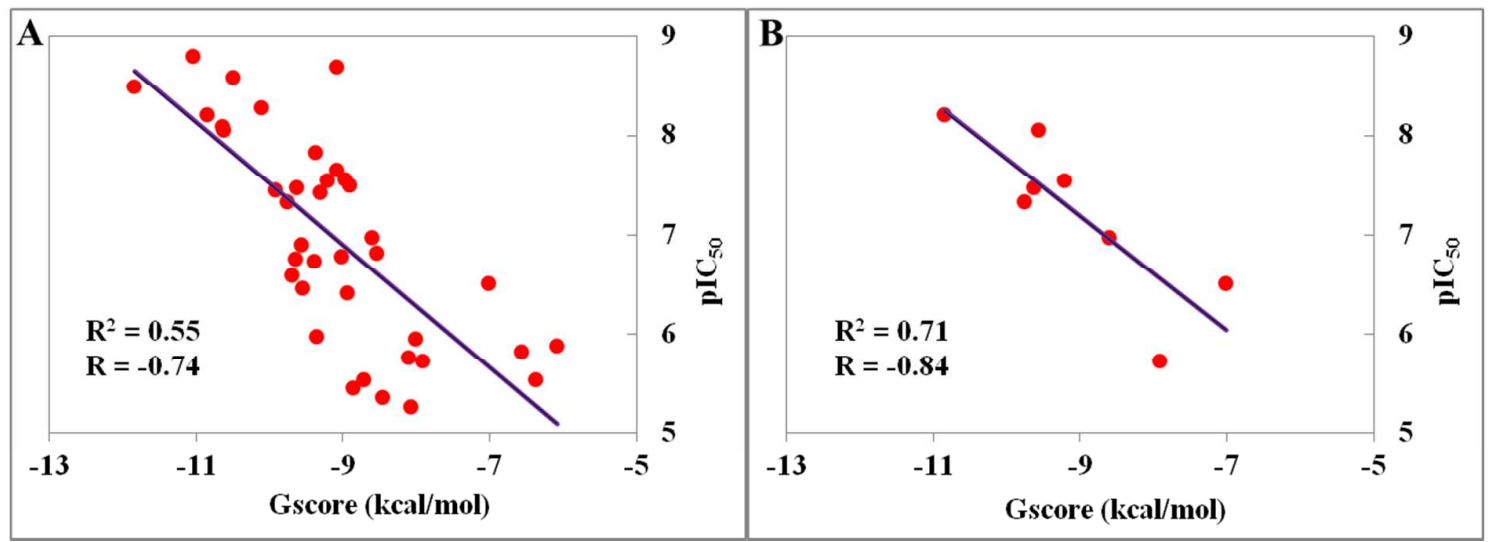

Figure S3. Linear relationship between the FLT3 $\mathrm{pIC}_{50} \mathrm{~s}$ and Gscores for (A) all pteridin-7(8H)-one compounds and three docked FLT3 type I inhibitors, and (B) compounds 1, 7, 11, 12, 18, 19, 22 and 23.

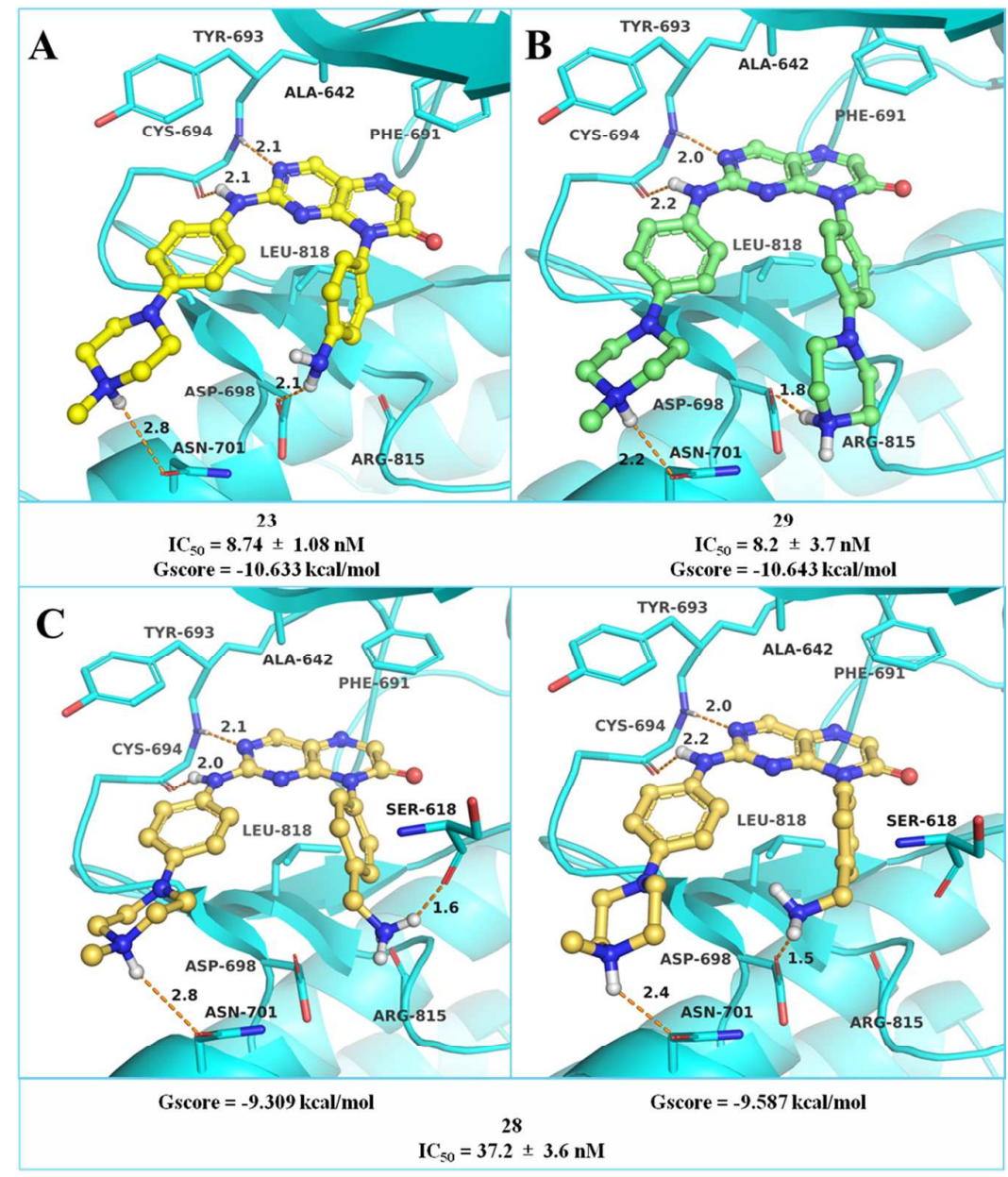

Figure S4. The predicted binding modes of compound 23(A), 28(C) and 29(B) with P-loop hidden for clarity. 


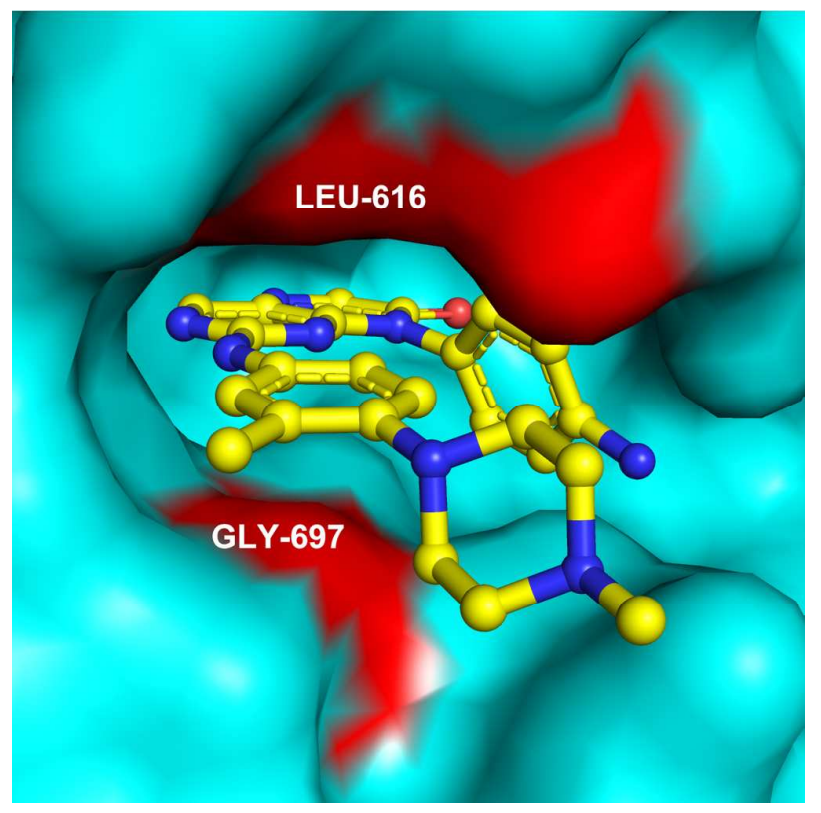

Figure S5. Compound 31 docked into the active site of the FLT3 homology model. The protein is shown as cyan surface, while the two residues of the solvent slot are highlighted in red color.

\section{Kinase Profiling Assay and Binding Constants $\left(K_{D} s\right)$ Assay.}

The kinase profiling assay and binding constants assay were conducted using the KINOMEscan platform (www.discoverx.com).

\section{Kinase assay.}

For most assays, kinase-tagged T7 phage strains were prepared in an E. coli host derived from the BL21 strain. E. coli were grown to log-phase and infected with $\mathrm{T} 7$ phage and incubated with shaking at $32^{\circ} \mathrm{C}$ until lysis. The lysates were centrifuged and filtered to remove cell debris. The remaining kinases were produced in HEK-293 cells and subsequently tagged with DNA for qPCR detection. Streptavidin-coated magnetic beads were treated with biotinylated small molecule ligands for 30 minutes at room temperature to generate affinity resins for kinase assays. The liganded beads were blocked with excess biotin and washed with blocking buffer (SeaBlock (Pierce), 1\% BSA, 0.05\% Tween 20, $1 \mathrm{mM}$ DTT) to remove unbound ligand and to reduce nonspecific binding. Binding reactions were assembled by combining kinases, liganded affinity beads, and test compounds in $1 \mathrm{x}$ binding buffer (20\% SeaBlock, 0.17x PBS, 0.05\% Tween 20, $6 \mathrm{mM}$ DTT). All reactions were performed in polystyrene 96-well plates in a final volume of $0.135 \mathrm{ml}$. The assay plates were incubated at room temperature with shaking for 1 hour and the affinity beads were washed with wash buffer (1x PBS, $0.05 \%$ Tween 20). The beads were then re-suspended in elution buffer (1x PBS, $0.05 \%$ Tween 20, $0.5 \mu \mathrm{M}$ non-biotinylated affinity ligand) and incubated at room temperature with shaking for 30 minutes. The kinase concentration in the eluates was measured by qPCR. 


\section{Compound handling.}

An 11-point 3-fold serial dilution of each test compound was prepared in 100\% DMSO at 100x final test concentration and subsequently diluted to $1 \mathrm{x}$ in the assay (final DMSO concentration $=1 \%$ ). Most $\mathrm{K}_{\mathrm{D}} \mathrm{s}$ were determined using a compound top concentration $=30,000 \mathrm{nM}$. If the initial $\mathrm{K}_{\mathrm{D}}$ determined was $<0.5 \mathrm{nM}$ (the lowest concentration tested), the measurement was repeated with a serial dilution starting at a lower top concentration. A $K_{D}$ value reported as $40,000 \mathrm{nM}$ indicates that the $\mathrm{K}_{\mathrm{D}}$ was determined to be $>30,000 \mathrm{nM}$.

\section{Binding Constants $\left(K_{D} s\right)$.}

Binding constants $\left(\mathrm{K}_{\mathrm{D}} \mathrm{s}\right)$ were calculated with a standard dose-response curve using the Hill equation:

Response $=$ Background $+\frac{\text { Signal }- \text { Background }}{1+\left(\mathrm{Kd}^{\text {Hill Slope }} / \text { Dose }^{\text {Hill Slope }}\right)}$

The Hill Slope was set to -1.Curves were fitted using a non-linear least square fit with the Levenberg-Marquardt algorithm.

Table S2 Kinase Selectivity of compound 31 against a panel of 468 kinases

\begin{tabular}{cc}
\hline Gene Symbol & Percent Control \\
\hline AAK1 & 18 \\
ABL1(E255K)-phosphorylated & 19 \\
ABL1(F317I)-nonphosphorylated & 95 \\
ABL1(F317I)-phosphorylated & 84 \\
ABL1(F317L)-nonphosphorylated & 92 \\
ABL1(F317L)-phosphorylated & 71 \\
ABL1(H396P)-nonphosphorylated & 35 \\
ABL1(H396P)-phosphorylated & 28 \\
ABL1(M351T)-phosphorylated & 31 \\
ABL1(Q252H)-nonphosphorylated & 22 \\
ABL1(Q252H)-phosphorylated & 26 \\
ABL1(T315I)-nonphosphorylated & 50 \\
ABL1(T315I)-phosphorylated & 6.3 \\
ABL1(Y253F)-phosphorylated & 42 \\
ABL1-nonphosphorylated & 42 \\
ABL1-phosphorylated & 32 \\
ABL2 & 63 \\
ACVR1 & 76 \\
ACVR1B & 96 \\
ACVR2A & 100
\end{tabular}


ACVR2B 100

ACVRL1 100

ADCK3 97

ADCK4 95

AKT1 100

AKT2 96

AKT3 100

ALK 54

ALK(C1156Y) $\quad 47$

ALK(L1196M) 73

AMPK-alpha1 13

AMPK-alpha2 $\quad 9.8$

ANKK1 89

ARK5 8.1

ASK1 100

ASK2 85

AURKA 1.4

AURKB 23

AURKC 26

AXL 21

BIKE 12

BLK 76

BMPR1A 100

BMPR1B 19

BMPR2 84

BMX 77

BRAF 92

BRAF(V600E) 77

BRK 63

BRSK1 71

BRSK2 100

BTK 6.6

BUB1 57

CAMK1 61

CAMK1B $\quad 37$

CAMK1D 46

CAMK1G 59

CAMK2A 78

CAMK2B 82

CAMK2D $\quad 76$

CAMK2G 68

CAMK4 100

CAMKK1 21

CAMKK2 19

CASK 71 


\begin{tabular}{|c|c|}
\hline $\mathrm{CDC} 2 \mathrm{~L} 1$ & 100 \\
\hline $\mathrm{CDC} 2 \mathrm{~L} 2$ & 100 \\
\hline CDC2L5 & 37 \\
\hline CDK11 & 75 \\
\hline CDK2 & 81 \\
\hline CDK3 & 93 \\
\hline CDK4 & 70 \\
\hline CDK4-cyclinD1 & 0.45 \\
\hline CDK4-cyclinD3 & 2.2 \\
\hline CDK5 & 84 \\
\hline CDK7 & 6.8 \\
\hline CDK8 & 82 \\
\hline CDK9 & 56 \\
\hline CDKL1 & 53 \\
\hline CDKL2 & 1.1 \\
\hline CDKL3 & 2.1 \\
\hline CDKL5 & 64 \\
\hline CHEK1 & 82 \\
\hline CHEK2 & 73 \\
\hline CIT & 78 \\
\hline CLK1 & 11 \\
\hline CLK2 & 20 \\
\hline CLK3 & 100 \\
\hline CLK4 & 8.6 \\
\hline CSF1R & 0.1 \\
\hline CSF1R-autoinhibited & 0 \\
\hline CSK & 95 \\
\hline CSNK1A1 & 96 \\
\hline CSNK1A1L & 98 \\
\hline CSNK1D & 94 \\
\hline CSNK1E & 100 \\
\hline CSNK1G1 & 81 \\
\hline CSNK1G2 & 89 \\
\hline CSNK1G3 & 100 \\
\hline CSNK2A1 & 11 \\
\hline CSNK2A2 & 1.7 \\
\hline CTK & 78 \\
\hline DAPK1 & 83 \\
\hline DAPK2 & 77 \\
\hline DAPK3 & 84 \\
\hline DCAMKL1 & 57 \\
\hline DCAMKL2 & 66 \\
\hline DCAMKL3 & 12 \\
\hline DDR1 & 9.8 \\
\hline DDR2 & 41 \\
\hline
\end{tabular}




\begin{tabular}{|c|c|}
\hline DLK & 90 \\
\hline DMPK & 37 \\
\hline DMPK2 & 100 \\
\hline DRAK1 & 2.4 \\
\hline DRAK2 & 21 \\
\hline DYRK1A & 79 \\
\hline DYRK1B & 62 \\
\hline DYRK2 & 78 \\
\hline EGFR & 90 \\
\hline EGFR(E746-A750del) & 77 \\
\hline EGFR(G719C) & 83 \\
\hline EGFR(G719S) & 79 \\
\hline EGFR(L747-E749del, A750P) & 84 \\
\hline EGFR(L747-S752del, P753S) & 86 \\
\hline EGFR(L747-T751del,Sins) & 70 \\
\hline EGFR(L858R) & 89 \\
\hline EGFR(L858R,T790M) & 57 \\
\hline EGFR(L861Q) & 62 \\
\hline EGFR(S752-I759del) & 80 \\
\hline EGFR(T790M) & 67 \\
\hline EIF2AK1 & 92 \\
\hline EPHA1 & 50 \\
\hline EPHA2 & 85 \\
\hline EPHA3 & 90 \\
\hline EPHA4 & 100 \\
\hline EPHA5 & 100 \\
\hline EPHA6 & 99 \\
\hline EPHA7 & 74 \\
\hline EPHA8 & 87 \\
\hline EPHB1 & 62 \\
\hline EPHB2 & 100 \\
\hline EPHB3 & 100 \\
\hline EPHB4 & 96 \\
\hline EPHB6 & 3.2 \\
\hline ERBB2 & 86 \\
\hline ERBB3 & 13 \\
\hline ERBB4 & 63 \\
\hline ERK1 & 100 \\
\hline ERK2 & 100 \\
\hline ERK3 & 100 \\
\hline ERK4 & 100 \\
\hline ERK5 & 100 \\
\hline ERK8 & 0.4 \\
\hline ERN1 & 57 \\
\hline FAK & 63 \\
\hline
\end{tabular}


$\begin{array}{ll}\text { FER } & 88\end{array}$

FES 78

FGFR1 78

FGFR2 43

FGFR3 100

FGFR3(G697C) 93

FGFR4 100

FGR 94

FLT1 66

FLT3 1.1

FLT3(D835H) 5.7

FLT3(D835V) 0

$\begin{array}{cc}\text { FLT3(D835Y) } & 5.1 \\ \text { FLT3(ITD) } & 0.35 \\ \text { FLT3(ITD,D835V) } & 0 \\ \text { FLT3(ITD,F691L) } & 0\end{array}$

FLT3(K663Q) 3.3

FLT3(N841I) $\quad 0$

FLT3(R834Q) 3.8

FLT3-autoinhibited $\quad 4.2$

FLT4 63

FRK 66

FYN 69

GAK 12

GCN2(Kin.Dom.2,S808G) 72

GRK1 92

GRK2 70

GRK3 79

GRK4 35

GRK7 $\quad 84$

GSK3A 99

GSK3B 97

HASPIN 92

HCK 96

HIPK1 2.1

HIPK2 1.2

HIPK3 8

HIPK4 54

HPK1 42

HUNK 100

ICK 17

IGF1R $\quad 86$

IKK-alpha $\quad 85$

IKK-beta 90

IKK-epsilon 93 
INSR $\quad 77$

INSRR 79

$\begin{array}{cc}\text { IRAK1 } & 0.3 \\ \text { IRAK3 } & 12 \\ \text { IRAK4 } & 81 \\ \text { ITK } & 58\end{array}$

JAK1(JH1domain-catalytic) 100

\begin{tabular}{|c|c|}
\hline JAK1(JH2domain-pseudokinase) & 0.35 \\
\hline JAK2(JH1domain-catalytic) & 4.6 \\
\hline JAK3(JH1 domain-catalytic) & 2.6 \\
\hline JNK1 & 66 \\
\hline JNK2 & 60 \\
\hline JNK3 & 57 \\
\hline KIT & 13 \\
\hline KIT(A829P) & 24 \\
\hline $\mathrm{KIT}(\mathrm{D} 816 \mathrm{H})$ & 16 \\
\hline KIT(D816V) & 2.7 \\
\hline KIT(L576P) & 1.7 \\
\hline KIT(V559D) & 7.7 \\
\hline KIT(V559D,T670I) & 33 \\
\hline KIT(V559D,V654A) & 55 \\
\hline KIT-autoinhibited & 3 \\
\hline LATS1 & 77 \\
\hline LATS2 & 41 \\
\hline LCK & 28 \\
\hline LIMK1 & 70 \\
\hline LIMK2 & 33 \\
\hline LKB1 & 95 \\
\hline LOK & 70 \\
\hline LRRK2 & 89 \\
\hline LRRK2(G2019S) & 73 \\
\hline LTK & 100 \\
\hline LYN & 70 \\
\hline LZK & 96 \\
\hline MAK & 50 \\
\hline MAP3K1 & 95 \\
\hline MAP3K15 & 85 \\
\hline MAP3K2 & 63 \\
\hline MAP3K3 & 50 \\
\hline MAP3K4 & 65 \\
\hline MAP4K2 & 70 \\
\hline MAP4K3 & 62 \\
\hline MAP4K4 & 100 \\
\hline MAP4K5 & 100 \\
\hline MAPKAPK2 & 100 \\
\hline
\end{tabular}




\begin{tabular}{|c|c|}
\hline MAPKAPK5 & 92 \\
\hline MARK1 & 23 \\
\hline MARK2 & 56 \\
\hline MARK3 & 45 \\
\hline MARK4 & 54 \\
\hline MAST1 & 68 \\
\hline MEK1 & 92 \\
\hline MEK2 & 47 \\
\hline MEK3 & 62 \\
\hline MEK4 & 100 \\
\hline MEK5 & 0.45 \\
\hline MEK6 & 77 \\
\hline MELK & 57 \\
\hline MERTK & 65 \\
\hline MET & 73 \\
\hline MET(M1250T) & 75 \\
\hline MET(Y1235D) & 100 \\
\hline MINK & 74 \\
\hline MKK7 & 94 \\
\hline MKNK1 & 71 \\
\hline MKNK2 & 11 \\
\hline MLCK & 72 \\
\hline MLK1 & 25 \\
\hline MLK2 & 92 \\
\hline MLK3 & 64 \\
\hline MRCKA & 91 \\
\hline MRCKB & 100 \\
\hline MST1 & 100 \\
\hline MST1R & 84 \\
\hline MST2 & 99 \\
\hline MST3 & 84 \\
\hline MST4 & 93 \\
\hline MTOR & 79 \\
\hline MUSK & 55 \\
\hline MYLK & 76 \\
\hline MYLK2 & 70 \\
\hline MYLK4 & 97 \\
\hline MYO3A & 94 \\
\hline MYO3B & 100 \\
\hline NDR1 & 51 \\
\hline NDR2 & 88 \\
\hline NEK1 & 93 \\
\hline NEK10 & 17 \\
\hline NEK11 & 99 \\
\hline NEK2 & 98 \\
\hline
\end{tabular}


NEK3 $\quad 81$

NEK4 79

NEK5 96

NEK6 85

NEK7 $\quad 80$

NEK9 93

NIK 93

NIM1 100

NLK 95

OSR1 79

p38-alpha 92

p38-beta 100

p38-delta 72

p38-gamma 73

PAK1 75

PAK2 89

PAK3 44

PAK4 68

PAK6 59

PAK7 49

PCTK1 4.8

PCTK2 28

PCTK3 85

PDGFRA 28

$\begin{array}{cc}\text { PDGFRB } & 0.95 \\ \text { PDPK1 } & 94 \\ \text { PFCDPK1(P.falciparum) } & 62 \\ \text { PFPK5(P.falciparum) } & 100 \\ \text { PFTAIRE2 } & 74 \\ \text { PFTK1 } & 89 \\ \text { PHKG1 } & 34 \\ \text { PHKG2 } & 43 \\ \text { PIK3C2B } & 95 \\ \text { PIK3C2G } & 95 \\ \text { PIK3CA } & 93 \\ \text { PIK3CA(C420R) } & 84 \\ \text { PIK3CA(E542K) } & 86 \\ \text { PIK3CA(E545A) } & 73 \\ \text { PIK3CA(E545K) } & 100 \\ \text { PIK3CA(H1047L) } & 91 \\ \text { PIK3CA(H1047Y) } & 91 \\ \text { PIK3CA(I800L) } & 100 \\ \text { PIK3CA(M1043I) } & 100 \\ \text { PIK3CA(Q546K) } & 92 \\ \text { PIK3CB } & 82\end{array}$




\begin{tabular}{|c|c|}
\hline PIK3CD & 68 \\
\hline PIK3CG & 75 \\
\hline PIK4CB & 100 \\
\hline PIKFYVE & 94 \\
\hline PIM1 & 90 \\
\hline PIM2 & 100 \\
\hline PIM3 & 100 \\
\hline PIP5K1A & 44 \\
\hline PIP5K1C & 67 \\
\hline PIP5K2B & 78 \\
\hline PIP5K2C & 19 \\
\hline PKAC-alpha & 100 \\
\hline PKAC-beta & 100 \\
\hline PKMYT1 & 93 \\
\hline PKN1 & 77 \\
\hline PKN2 & 85 \\
\hline PKNB(M.tuberculosis) & 4.9 \\
\hline PLK1 & 97 \\
\hline PLK2 & 79 \\
\hline PLK3 & 88 \\
\hline PLK4 & 29 \\
\hline PRKCD & 88 \\
\hline PRKCE & 90 \\
\hline PRKCH & 100 \\
\hline PRKCI & 69 \\
\hline PRKCQ & 89 \\
\hline PRKD1 & 96 \\
\hline PRKD2 & 100 \\
\hline PRKD3 & 90 \\
\hline PRKG1 & 100 \\
\hline PRKG2 & 80 \\
\hline PRKR & 57 \\
\hline PRKX & 80 \\
\hline PRP4 & 83 \\
\hline PYK2 & 53 \\
\hline QSK & 86 \\
\hline RAF1 & 94 \\
\hline RET & 57 \\
\hline RET(M918T) & 36 \\
\hline RET(V804L) & 42 \\
\hline RET(V804M) & 43 \\
\hline RIOK1 & 23 \\
\hline RIOK2 & 71 \\
\hline RIOK3 & 34 \\
\hline RIPK1 & 92 \\
\hline
\end{tabular}




\begin{tabular}{|c|c|}
\hline RIPK2 & 97 \\
\hline RIPK4 & 93 \\
\hline RIPK5 & 88 \\
\hline ROCK1 & 93 \\
\hline ROCK2 & 71 \\
\hline ROS1 & 32 \\
\hline RPS6KA4(Kin.Dom.1-N-terminal) & 97 \\
\hline RPS6KA4(Kin.Dom.2-C-terminal) & 82 \\
\hline RPS6KA5(Kin.Dom.1-N-terminal) & 100 \\
\hline RPS6KA5(Kin.Dom.2-C-terminal) & 100 \\
\hline RSK1(Kin.Dom.1-N-terminal) & 98 \\
\hline RSK1(Kin.Dom.2-C-terminal) & 91 \\
\hline RSK2(Kin.Dom.1-N-terminal) & 58 \\
\hline RSK2(Kin.Dom.2-C-terminal) & 90 \\
\hline RSK3(Kin.Dom.1-N-terminal) & 86 \\
\hline RSK3(Kin.Dom.2-C-terminal) & 97 \\
\hline RSK4(Kin.Dom.1-N-terminal) & 92 \\
\hline RSK4(Kin.Dom.2-C-terminal) & 89 \\
\hline S6K1 & 45 \\
\hline SBK1 & 30 \\
\hline SGK & 46 \\
\hline $\operatorname{SgK} 110$ & 100 \\
\hline SGK2 & 86 \\
\hline SGK3 & 59 \\
\hline SIK & 68 \\
\hline SIK2 & 33 \\
\hline SLK & 8 \\
\hline SNARK & 1.6 \\
\hline SNRK & 95 \\
\hline SRC & 25 \\
\hline SRMS & 84 \\
\hline SRPK1 & 35 \\
\hline SRPK2 & 96 \\
\hline SRPK3 & 52 \\
\hline STK16 & 2.4 \\
\hline STK33 & 98 \\
\hline STK35 & 96 \\
\hline STK36 & 5 \\
\hline STK39 & 53 \\
\hline SYK & 65 \\
\hline TAK1 & 45 \\
\hline TAOK1 & 77 \\
\hline TAOK2 & 100 \\
\hline TAOK3 & 84 \\
\hline TBK1 & 38 \\
\hline
\end{tabular}




\begin{tabular}{|c|c|}
\hline TEC & 97 \\
\hline TESK1 & 59 \\
\hline TGFBR1 & 84 \\
\hline TGFBR2 & 16 \\
\hline TIE1 & 55 \\
\hline TIE2 & 84 \\
\hline TLK1 & 37 \\
\hline TLK2 & 41 \\
\hline TNIK & 79 \\
\hline TNK1 & 34 \\
\hline TNK2 & 95 \\
\hline TNNI3K & 85 \\
\hline TRKA & 4.7 \\
\hline TRKB & 45 \\
\hline TRKC & 51 \\
\hline TRPM6 & 99 \\
\hline TSSK1B & 84 \\
\hline TSSK3 & 78 \\
\hline TTK & 24 \\
\hline TXK & 99 \\
\hline I1domain-catalytic) & 27 \\
\hline lomain-pseudokinase) & 2.9 \\
\hline TYRO3 & 84 \\
\hline ULK1 & 44 \\
\hline ULK2 & 69 \\
\hline ULK3 & 16 \\
\hline VEGFR2 & 31 \\
\hline VPS34 & 46 \\
\hline VRK2 & 96 \\
\hline WEE1 & 100 \\
\hline WEE2 & 97 \\
\hline WNK1 & 87 \\
\hline WNK2 & 92 \\
\hline WNK3 & 92 \\
\hline WNK4 & 95 \\
\hline YANK1 & 75 \\
\hline YANK2 & 100 \\
\hline YANK3 & 85 \\
\hline YES & 56 \\
\hline YSK1 & 51 \\
\hline YSK4 & 27 \\
\hline ZAK & 33 \\
\hline ZAP70 & 80 \\
\hline
\end{tabular}


Table S3 S-score Table for compound 31

\begin{tabular}{cccccc}
\hline $\begin{array}{c}\text { Compound } \\
\text { Name }\end{array}$ & $\begin{array}{c}\text { Selectivity } \\
\text { Score Type }\end{array}$ & $\begin{array}{c}\text { Number of } \\
\text { Hits }\end{array}$ & $\begin{array}{c}\text { Number of } \\
\text { Non-Mutant } \\
\text { Kinases }\end{array}$ & $\begin{array}{c}\text { Screening } \\
\text { Concentration } \\
(\mathrm{nM})\end{array}$ & $\begin{array}{c}\text { Selectivity } \\
\text { Score }\end{array}$ \\
\hline $\mathbf{3 1}$ & $\mathrm{S}(35)$ & 78 & 403 & 1000 & 0.194 \\
$\mathbf{3 1}$ & $\mathrm{S}(10)$ & 34 & 403 & 1000 & 0.084 \\
$\mathbf{3 1}$ & $\mathrm{S}(1)$ & 7 & 403 & 1000 & 0.017 \\
\hline
\end{tabular}


The in vitro enzymatic selectivity against FLT3 over EGFR.

Table S4 In vitro enzymatic inhibitory activities and enzyme selectivity of Pteridin-7(8H)-one compounds.

\begin{tabular}{|c|c|c|c|}
\hline Comp. & $\begin{array}{l}\text { In vitro Enzyme FLT3 Inhibitory Activity } \\
\qquad\left(\mathrm{IC}_{50}, \mathrm{nM}\right)\end{array}$ & $\begin{array}{l}\text { In vitro Enzyme EGFR }{ }^{\mathrm{WT}} \text { Inhibitory Activity }{ }^{\mathrm{a}} \\
\qquad\left(\mathrm{IC}_{50}, \mathrm{nM}\right)\end{array}$ & $\begin{array}{c}\text { Enzyme Selectivity } \\
\text { EGFR/FLT3 }\end{array}$ \\
\hline 1 & $312 \pm 11$ & $1.21 \pm 0.27$ & 0.0039 \\
\hline 2 & $1528 \pm 444$ & $15.3 \pm 6.9$ & 0.01 \\
\hline 3 & $1056 \pm 178$ & $15.7 \pm 3.6$ & 0.015 \\
\hline 4 & $1747 \pm 10$ & $14.5 \pm 7.9$ & 0.0083 \\
\hline 5 & $2958 \pm 1166$ & $12.1 \pm 0.7$ & 0.0041 \\
\hline 6 & $5449 \pm 1901$ & $12.5 \pm 2.9$ & 0.0023 \\
\hline 7 & $1869 \pm 278$ & $13.8 \pm 4.8$ & 0.0074 \\
\hline 8 & $2920 \pm 1061$ & $12.8 \pm 2.21$ & 0.0044 \\
\hline 9 & $3589 \pm 764$ & $47.10 \pm 5.74$ & 0.013 \\
\hline 10 & $1136 \pm 338$ & $6.95 \pm 0.89$ & 0.0061 \\
\hline 11 & $47 \pm 4$ & $1100 \pm 167$ & 23 \\
\hline 12 & $108 \pm 20$ & $5129 \pm 2697$ & 47 \\
\hline 13 & $165 \pm 36$ & $>10000$ & 61 \\
\hline 14 & $384 \pm 26$ & $373.5 \pm 186.3$ & 0.97 \\
\hline 15 & $173 \pm 36$ & $5585 \pm 2983$ & 32 \\
\hline 16 & $4413 \pm 26$ & $>10000$ & 2.3 \\
\hline 17 & $184 \pm 32$ & $>10000$ & 54 \\
\hline 18 & $34 \pm 4$ & $>10000$ & 294 \\
\hline 19 & $29 \pm 2$ & $>10000$ & 345 \\
\hline 20 & $1317 \pm 148$ & $5.29 \pm 1.7$ & 0.0040 \\
\hline 21 & $153 \pm 27$ & $>10000$ & 65 \\
\hline
\end{tabular}




\begin{tabular}{|c|c|c|c|}
\hline 22 & $6.13 \pm 0.37$ & n.d. ${ }^{b}$ & n.d. \\
\hline 23 & $8.74 \pm 0.76$ & n.d. & n.d. \\
\hline 24 & $127 \pm 25$ & n.d. & n.d. \\
\hline 25 & $255 \pm 59$ & n.d. & n.d. \\
\hline 26 & $22.2 \pm 3.6$ & n.d. & n.d. \\
\hline 27 & $32.0 \pm 9.6$ & n.d. & n.d. \\
\hline 28 & $37.2 \pm 2.4$ & n.d. & n.d. \\
\hline 29 & $8.2 \pm 2.6$ & n.d. & n.d. \\
\hline 30 & $347 \pm 50$ & n.d. & n.d. \\
\hline 31 & $1.56 \pm 0.04$ & $>10000^{c}$ & 6410 \\
\hline 32 & $3.19 \pm 0.73$ & n.d. & n.d. \\
\hline 33 & $5.24 \pm 0.03$ & n.d. & n.d. \\
\hline 34 & $27.7 \pm 1.1$ & n.d. & n.d. \\
\hline 35 & $35.8 \pm 2.8$ & n.d. & n.d. \\
\hline $\mathrm{AC} 220$ & $4.2 \pm 0.3^{\mathrm{d}}$ & n.d. & n.d. \\
\hline MLN518 & $220^{\mathrm{e}}$ & n.d. & n.d. \\
\hline Stauroporine & $0.2 \pm 0.02$ & n.d. & n.d. \\
\hline
\end{tabular}

a. The data come from the paper (J. Med. Chem. 2013, 56 (20), 7821-7837.)

b. Not Determined.

c. We test it by the same method in the paper mentioned above.

d. Data from the paper (Blood 2009, 114 (14), 2984-2992.)

e. Data from the paper (Cancer Cell 2002, 1(5):421-432.) 
Compound 23 inhibited FLT3 auto-phosphorylation and the phosphorylation of downstream signaling effectors STAT5, ERK, and AKT in 2 hours.

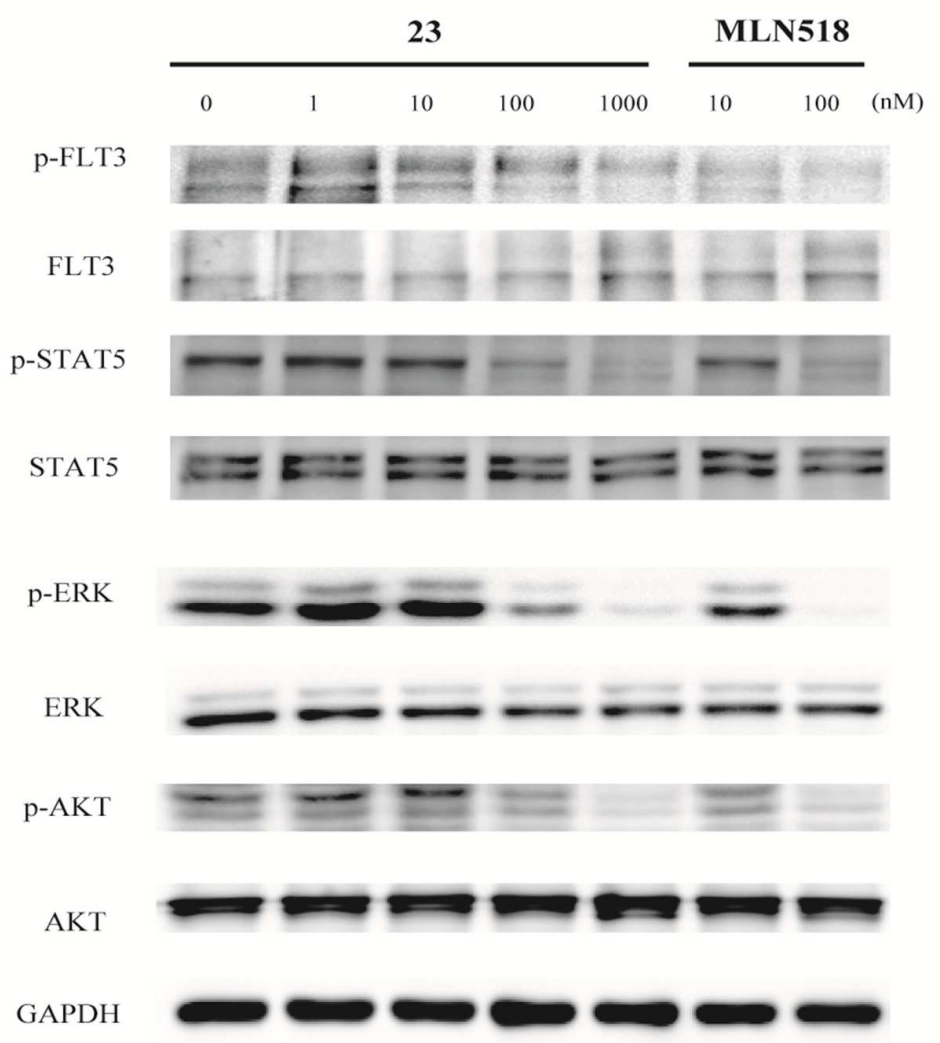

Figure S6. After 2 hours treatment, compound 23 inhibited FLT3 auto-phosphorylation and the phosphorylation of downstream signaling effectors STAT5, ERK, and AKT in MV4-11 cells. 


\section{Compound 23 induce apoptosis and cell cycle arrest in MV4-11 after $48 \mathrm{~h}$}

\section{treatment.}

A
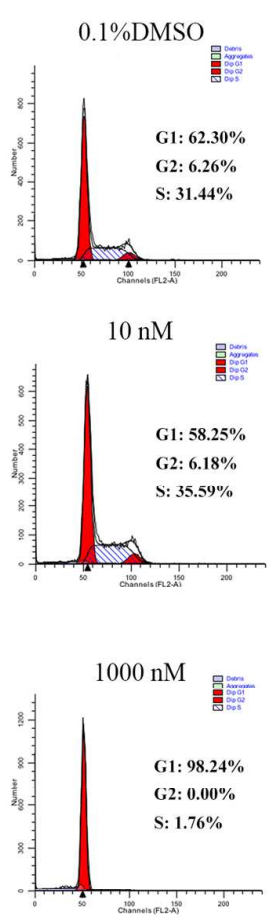
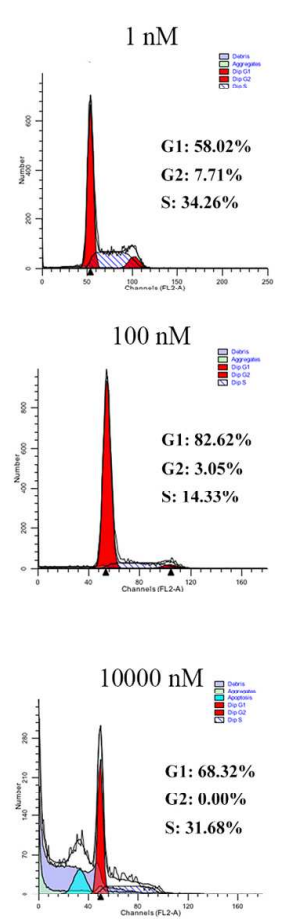

B
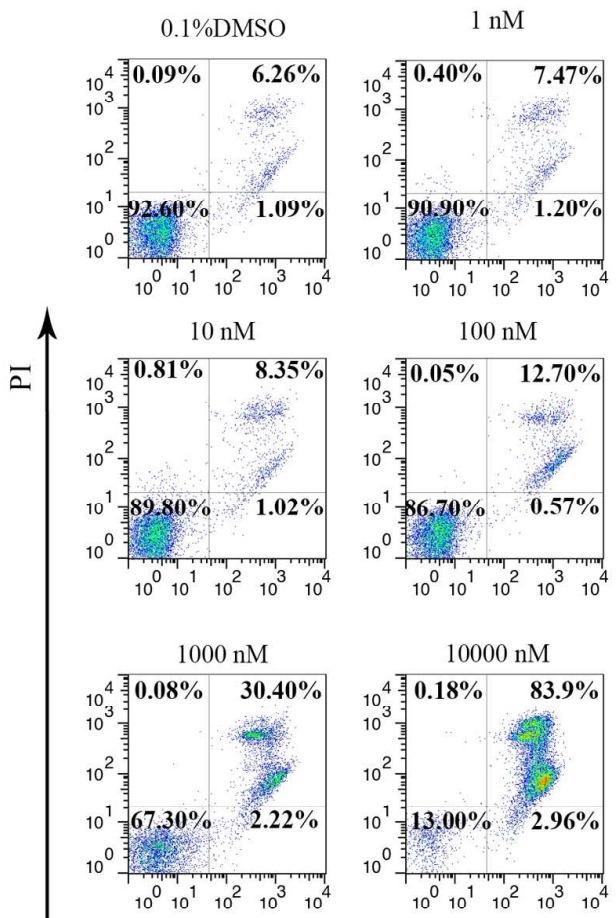

Annexin V

Figure S7. After 48 hours treatment, compound 23 induced dose-dependent $G_{0} / G_{1}$ phase cell cycle arrest (A) and apoptosis (B) in MV4-11 cells. 
In vivo effecacy of compound 31 against MV4-11 tumor xenografts via intraperitoneal administration.

A

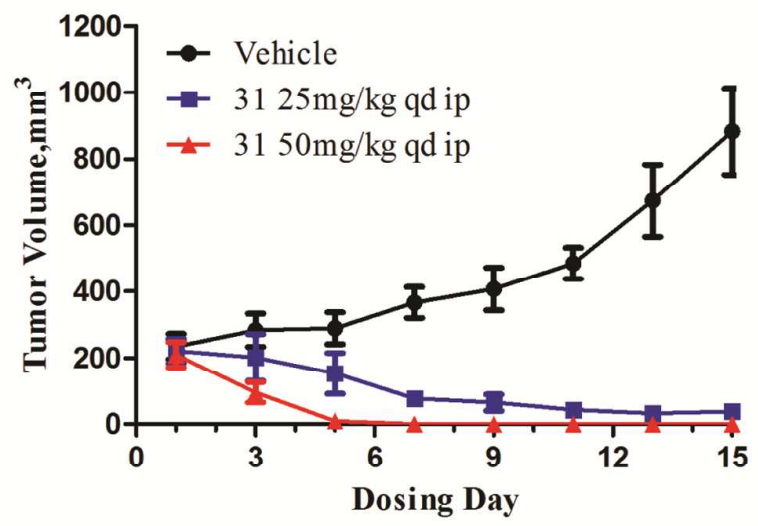

B

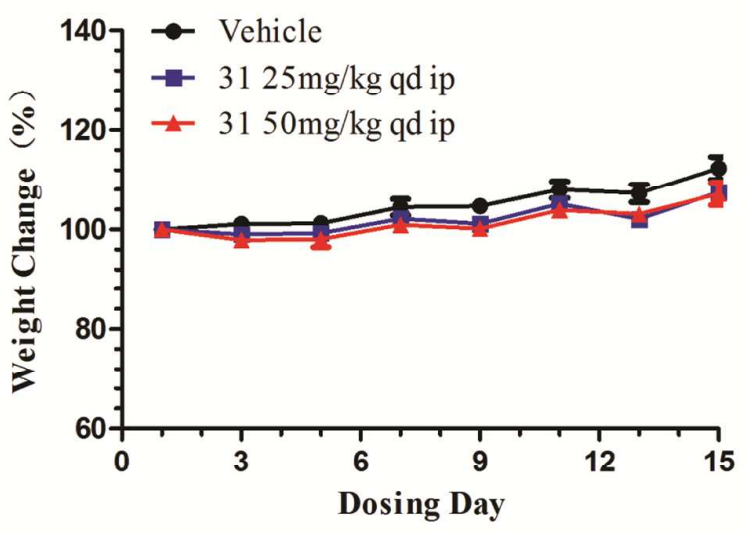

Figure S8. In vivo effects of compound 31 against MV4-11 tumor xenografts via intraperitoneal administration. (A) Compound 31 was administered intraperitoneally at $25,50 \mathrm{mg} / \mathrm{kg} / \mathrm{d}$. Data are shown as mean \pm SEM. (B) Body weight change in mouse xenograft model for each daily dosing group. 


\section{Model Validation.}
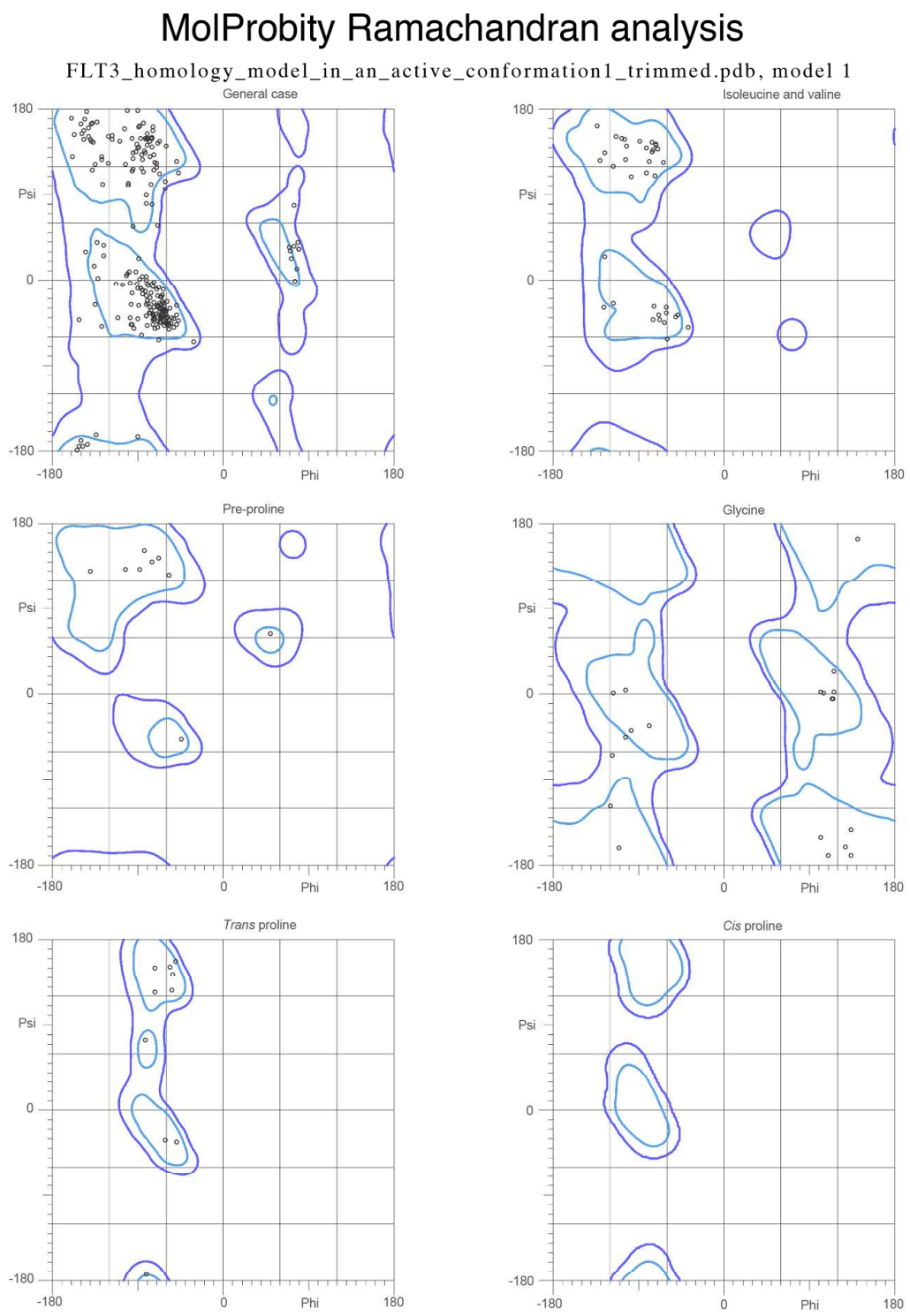

$91.5 \%(280 / 306)$ of all residues were in favored (98\%) regions. $100.0 \%(306 / 306)$ of all residues were in allowed (>99.8\%) regions.

There were no outliers.

http://kinemage. biochem.duke.edu

Lovell, Davis, et al. Proteins 50:437 (2003)

Figure S9. Ramachandran plot of the FLT3 homology model in an active conformation. 


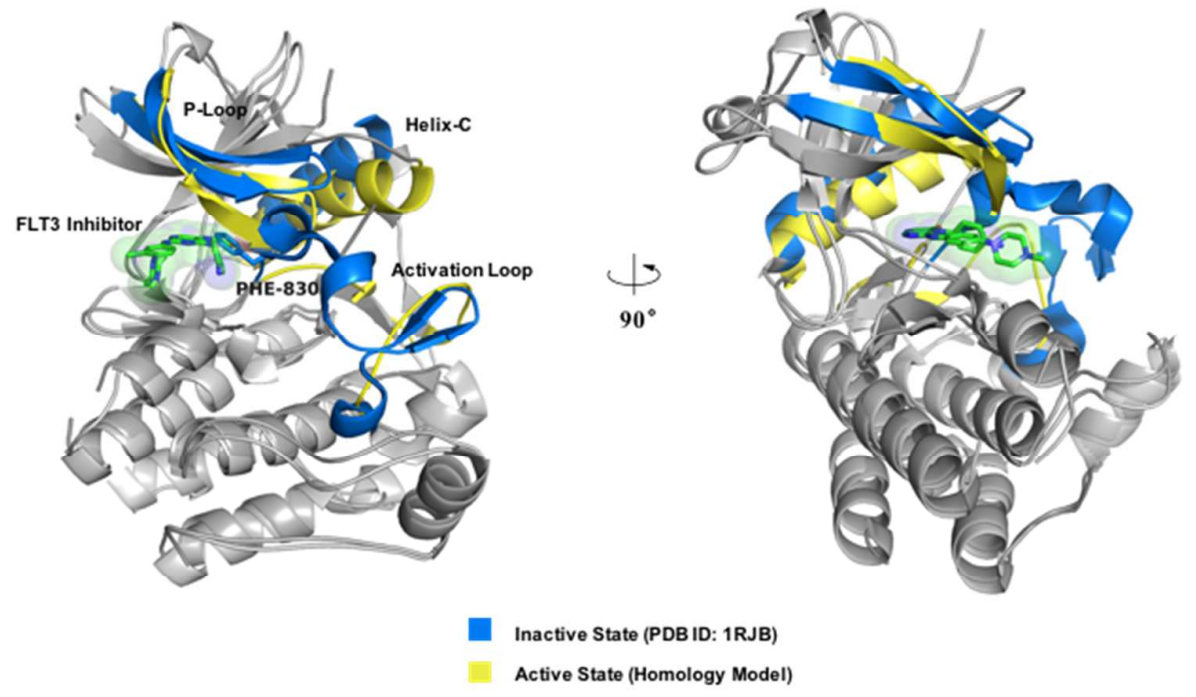

Figure S10. Active and inactive conformations of FLT3. The template protien of FLT3 homology model in an active conformation is a c-KIT kinase (PDB ID: 1PKG).

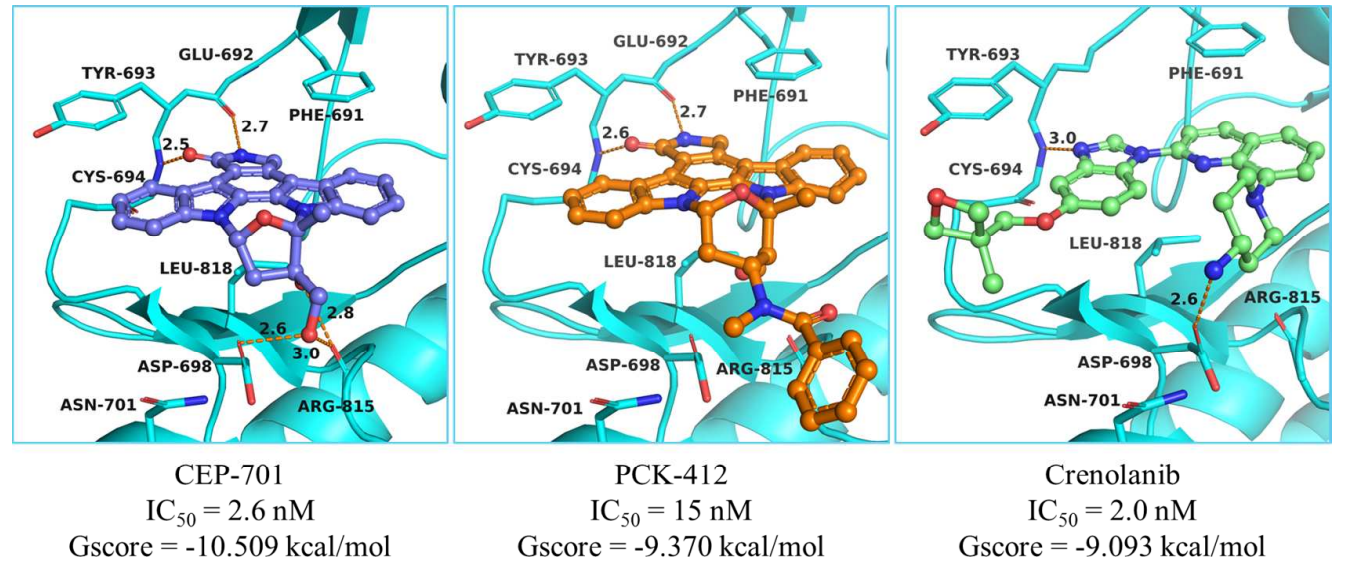

Figure S11. The predicted binding modes, the corresponding FLT3 $\mathrm{IC}_{50}$ values and binding energies of the three known FLT3 type I inhibitors. P-loop is hid for clarity. The $\mathrm{IC}_{50}$ values of CEP-701 and PCK-412 from Blood 2009, 114 (14): 2984-2992. The $\mathrm{IC}_{50}$ value of Crenolanib from Blood 2014,123(1):94-100. 